\title{
Using the Millennium II simulation to test CDM predictions for the structure of massive galaxies
}

\author{
Andrew P. Cooper ${ }^{1} \dagger$, Guinevere Kauffmann ${ }^{1}$, Jing Wang ${ }^{1}$ \\ and Simon D. M. White ${ }^{1}$ \\ ${ }^{1}$ Max Planck Institut für Astrophysik, Karl-Schwarzschild-Str. 1, 85748 Garching, Germany \\ email: acooper@nao.cas.cn
}

\begin{abstract}
We have combined the semi-analytic galaxy formation model of Guo et al. (2011) with a novel particle-tagging technique to predict galaxy surface brightness profiles in a representative sample of $\sim 1900$ massive dark matter haloes $\left(10^{12}-10^{14} \mathrm{M}_{\odot}\right)$ from the Millennium II $\Lambda$ CDM $N$ body simulation. We focus on the outer regions of galaxies and stars accreted in mergers. Our simulations cover scales from the stellar haloes of Milky Way-like galaxies to the 'cD envelopes' of groups and clusters, and resolve low surface brightness substructure such as the tidal streams of dwarf galaxies. We find that the spatial distribution of stars in low surface brightness regions is tightly correlated with DM halo mass and that collisionless merging during the hierarchical assembly of galaxies largely determines the structure of spheroidal stellar components. Our $\Lambda \mathrm{CDM}$ model agrees well with the available data.
\end{abstract}

Keywords. galaxies: elliptical and lenticular, $\mathrm{cD}$, galaxies: kinematics and dynamics, galaxies: structure, methods: n-body simulations

\section{Introduction}

Galaxies in a cold dark matter (CDM) universe are assembled both by in situ star formation and by galactic accretion and merging (White \& Rees 1978). The balance between these two channels affects how stars and chemical elements are distributed within present-day galaxies. In particular, accretion and merger events are thought to supply most of the stars that contribute to the outer regions of galaxies (e.g. the stellar halo of the Milky Way; Searle \& Zinn 1978). The diffuse intracluster light around massive cluster central galaxies may have a similar origin and account for a large fraction of the total stellar mass. Future surveys such as LSST will make possible detailed studies of low surface brightness regions in most massive galaxies at low redshift and thereby provide new ways to test models of galaxy evolution.

The semi-analytic approach treats the fundamental astrophysical processes involved in galaxy formation as a self-consistent system of rate equations, which describe the transfer of mass between different phases (White \& Frenk 1991, Kauffmann, White \& Guiderdoni 1993, Cole et al. 2000). Given cosmological initial conditions and a model for the hierarchical clustering of dark matter, such models provide ab initio predictions for the properties of an entire $\Lambda \mathrm{CDM}$ galaxy population. They allow an efficient exploration of parameter space, leading to a more intuitive understanding of the interplay between different processes, and produce results that can be compared statistically to observations

$\dagger$ Present address: National Astronomical Observatories, Chinese Academy of Scienes, 20A Datun Rd., Beijing 100012, P.R. China 
from large galaxy surveys. Our aim is to use the model of Guo et al. (2011) to make statistical predictions for the distribution of accreted stars in massive galaxies.

\section{Model}

Structural predictions from semi-analytic models are currently limited to 1D radial profiles. We have developed a new technique which we call 'particle tagging' (described in detail in Cooper et al. 2010; see also Bullock \& Johnston 2005) to extend these predictions to all six dimensions of phase space, as in an $N$ body simulation. We 'tag' a set of DM particles in the Millennium II simulation (Boylan-Kolchin et al. 2009) for every distinct stellar population (defined by a single age and metallicity) that forms according to the Guo et al. (2011) model. Particles are chosen according to their binding energy at the time when their associated population forms, such that they initially occupy a region of phase space appropriate for new stars after dissipative collapse. The phase space evolution of the tagged particles has already been computed, so we use it as a proxy for that of their associated stars, assuming that the gravity of the stars does not alter the evolution of the system. This is likely to be a poor approximation in the centres of massive galaxies, but adequate for accreted stars in their outer regions.

\section{Results}

Figure 1 shows nine examples of projected stellar mass surface density distributions, from the $\sim 1900$ massive DM haloes in the Millennium II simulation to which we applied our particle tagging model. We have removed satellite galaxies to show only the light associated with the central galaxy. Galaxies labelled A, E and I are associated with haloes of $M_{200} \sim 10^{12.5} \mathrm{M}_{\odot}$ while the rest are the central galaxies of massive clusters, $M_{200} \sim 10^{13.5} \mathrm{M}_{\odot} \dagger$. Black pixels correspond to a surface density of $\sim 10^{3} \mathrm{M}_{\odot} \mathrm{kpc}^{-2}$ and white pixels to $\sim 10^{9} \mathrm{M}_{\odot} \mathrm{kpc}^{-2}$. The overall light distribution is elongated in most cases, reflecting the triaxial nature of DM haloes. The brightest regions (out to $R \sim 15 \mathrm{kpc}$ ) are dominated by stars formed in situ, while the outer regions are dominated by accreted stars. Streams and shells from recently disrupted companion galaxies are clearly visible.

Figure 2 shows the median surface density profiles of our simulated galaxies obtained by stacking them in bins of $M_{200}$. The shape of the median profile has a tight correlation with $M_{200}$. In low mass haloes (comparable to the Milky Way and M31; Li \& White 2008) the profile has a clear inflection to a shallower outer slope at $\sim 15 \mathrm{kpc}$; in more massive haloes it is close to a powerlaw over the range $10<R<100 \mathrm{kpc}$, falling off gradually at larger radius. The trends in our simulations are comparable to those observed, both for individual galaxies and stacks, a number of which we compare in Figure 2.

The behaviour of the average profile can be easily understood by splitting it into in situ and accreted components, as has been emphasised by a number of recent studies (Abadi et al. 2006, Cooper et al. 2010, Oser et al. 2010). Both components can be well-described by Sersic profiles, but they scale differently with $M_{200}$ according both to the mass-dependent efficiency of star formation (e.g. Moster et al. 2010) and the mass spectrum, accretion time and orbits of hierarchical progenitors. This is a natural outcome of galaxy formation in CDM (e.g. Cole et al. 2000, Purcell et al. 2007). Our a priori $6 \mathrm{D}$ model of galactic structure is constrained to match the observed galaxy mass function and agrees well with other galaxy population data, and its predictions can therefore be compared directly to observations. The particle tagging technique has

$\dagger M_{200}$ is the mass within a radius enclosing a mean density 200 times the critical density. 


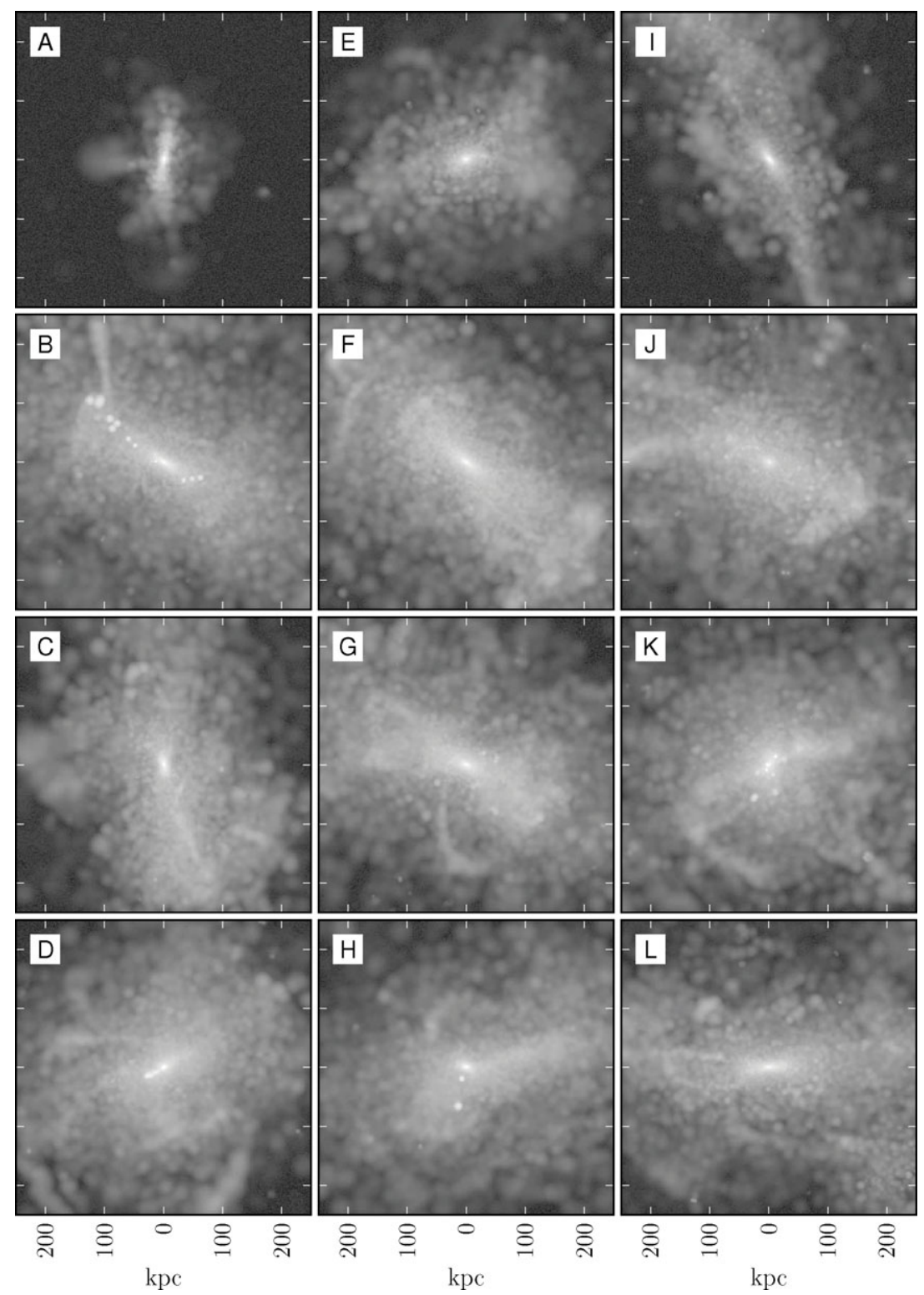

Figure 1. Images of nine of our simulated central galaxies, $250 \times 250 \mathrm{kpc}$. The logarithmic grey scale corresponds to stellar mass surface density over the range $3<\log _{10} \Sigma / \mathrm{M}_{\odot} \mathrm{kpc}^{-2}<9$. The first row (labels A, E, I) shows three galaxies with $M_{\star} \sim 10^{11} \mathrm{M}_{\odot}$ and $\mathrm{DM}$ halo mass $M_{200} \sim 10^{13} \mathrm{M}_{\odot}$. The other panels show the central galaxies of cluster-mass haloes $M_{200} \sim 10^{14} \mathrm{M}_{\odot}$. Satellite galaxies are not shown. Simulation particles have been smoothed with an $N=64$ cubic spline kernel; the remaining lumpiness in these images is due to shot noise in the particle distribution.

a number of interesting applications that we will present in forthcoming papers, include stellar population gradients in early-type galaxies; the kinematics of diffuse light; the effects of interactions on the structure of satellite galaxies and environmental trends; the frequency of tidal features and their correlations with other galaxy properties; and the intracluster light of massive clusters. 


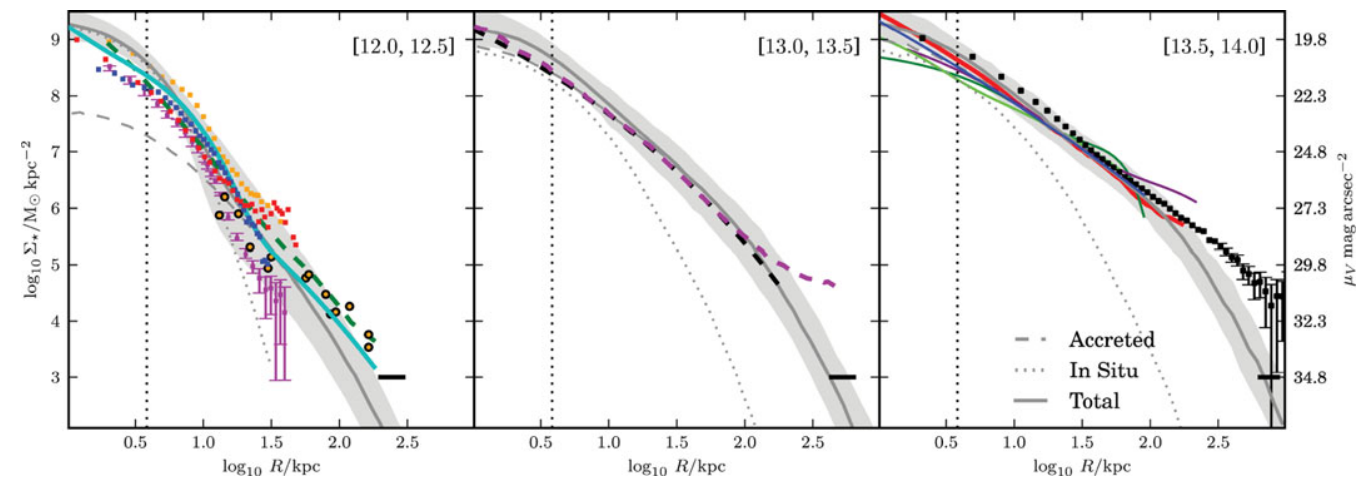

Figure 2. Median surface density profiles (shaded region shows 10-90 percentile range) for stacks of simulated galaxies in three bins of $\log _{10} M_{200}$, compared with observations. Simulated profiles are split accreted (dashed) and in situ (dotted) contributions. Black bar shows the range of virial radii $R_{200}$ in each mass bin. Data is taken from the following authors. Left: Barker et al. (2009), Gilbert et al. (2009), Courteau et al. (2011), Font et al. (2011; GIMIC simulations), Barker et al. (2012), Bakos \& Trujillo (2012). Centre: van Dokkum et al.(2010), Tal \& van Dokkum (2011). Right: Zibetti et al.(2005), Seigar et al. (2007), Kormendy et al. (2009).

\section{References}

Abadi, M. G., Navarro, J. F., \& Steinmetz, M. 2006, MNRAS, 365, 747

Barker, M. K., Ferguson, A. M., Irwin, M., et al. 2009, AJ, 138, 1469

Barker, M. K., Ferguson, A. M., Irwin, M. J., et al. 2012, MNRAS, 419, 1489

Bakos, J. \& Trujillo, I. 2012, ApJ, submitted (arXiv:1204.3082)

Boylan-Kolchin, M., Springel, V., White, S. D. M., \& Jenkins, A., Lemson G. 2009, MNRAS, 398,1150

Bullock, J. S. \& Johnston, K. V. 2005, ApJ, 635, 931

Cole, S. 1991, ApJ, 367, 45

Cole, S., Lacey, C. G., Baugh, C. M., \& Frenk, C. S. 2000, MNRAS, 319, 168

Cooper, A. P., et al. 2010, MNRAS, 406, 744

Courteau, S., et al. 2011, ApJ, 739, 20

Font, A. S., et al. 2011, MNRAS, 416, 2802

Gilbert, K. M., Font, A. S., Johnston, K. V., \& Guhathakurta, P. 2009, ApJ, 701, 776

Guo, Q., et al. 2011, MNRAS, 413, 101

Kauffmann, G., White, S. D. M., \& Guiderdoni, B. 1993, MNRAS, 264, 201

Kormendy, J., Fisher, D. B., Cornell, M. E., \& Bender, R. 2009, ApJS, 182, 216

Li, Y.-S. \& White, S. D. M. 2008, MNRAS, 384, 1459

Moster, B. P., et al. 2010, ApJ, 710, 903

Searle, L. \& Zinn, R. 1978, ApJ, 225, 357

Seigar, M. S., Graham, A. W., \& Jerjen, H. 2007, MNRAS, 378, 1575

Somerville, R. S. \& Primack J. R. 1999, MNRAS, 310, 1087

Tal, T. \& van Dokkum, P. G. 2011, ApJ, 731, 89

van Dokkum, P. G., et al. 2010, ApJ, 709, 1018

White, S. D. M. \& Frenk, C. S. 1991, ApJ, 379, 52

White, S. D. M. \& Rees, M. J. 1978, MNRAS, 183, 341

Zibetti, S., White, S. D. M., Schneider, D. P., \& Brinkmann, J. 2005, MNRAS, 358, 949 